\title{
Information Transfer and Biological Significance of Neoplastic Exosomes in the Tumor Microenvironment of Osteosarcoma
}

This article was published in the following Dove Press journal: OncoTargets and Therapy

\author{
Feifei Pu $\mathbb{1}^{1, *}$ \\ Fengxia Chen ${ }^{2, *}$ \\ Zhicai Zhang' \\ Jianxiang Liu' \\ Zengwu Shao' \\ 'Department of Orthopedics, Union \\ Hospital, Tongji Medical College, \\ Huazhong University of Science and \\ Technology, Wuhan, Hubei, People's \\ Republic of China; ${ }^{2}$ Department of \\ Radiation and Medical Oncology, \\ Zhongnan Hospital, Wuhan University, \\ Wuhan, Hubei, People's Republic of \\ China
}

*These authors contributed equally to this work
Correspondence: Zengwu Shao Email 1985XH0536@hust.edu.cn

\begin{abstract}
Osteosarcoma is a highly invasive kind of malignant bone tumor. Exosomes are a type of extracellular vesicles that play an important role in intercellular communication in the microenvironment. Tumor cell progression is promoted through the interaction between exosomes and cells in the microenvironment (including immune cells, mesenchymal cells, and endothelial cells) during tumor development. Neoplastic exosomes can carry a variety of biological information molecules, such as proteins, lipids, and nucleic acids. These molecules play an important clinical role, not only being able domesticate the recipient cells but also being recognized as tumor specific markers. At the same time, exosomes secreted by osteosarcoma can also cooperate with antigen-presenting cells to activate the body's immune response and then to exert anti-tumor effects. Studies on exosomes may be a breakthrough in the search for a new osteosarcoma treatment. In this study, we review the role of neoplastic exosomes in the osteosarcoma microenvironment, summarize their potential as tumor markers, and investigate their clinical application prospects.
\end{abstract}

Keywords: osteosarcoma, exosome, diagnosis, treatment, drug carrier, antitumor drug

\section{Introduction}

Osteosarcoma is a kind of malignant bone tumor that occurs in children and adolescents. Due to the treatment using neoadjuvant chemotherapy, the prognosis and survival rate of patients have been significantly improved. However, osteosarcoma is prone to metastasis, and its 5-year survival rate is still lower than $60 \%{ }^{1}$ In recent years, significant research achievements have been obtained on the pathogenesis of tumors regulated by exosomes. ${ }^{2}$ Studies have shown that osteosarcoma cells can secrete exosomes, promote tumor growth, metastasis, angiogenesis, and evade immune surveillance by regulating the tumor microenvironment. ${ }^{3,4}$ Exosomes can transport various proteins, signaling pathway factors, mRNA, DNA, microRNAs (miRNAs), adenosine triphosphatase (ATPase) and phosphoglycerate kinase (PGKL) enzymes, transcription factors, adhesion factors, heat shock proteins, cytoskeleton proteins, lipid rafts, membrane transporters, among others, and they play important roles in exchanging intercellular information and in changing the function of recipient cells. ${ }^{4}$ Studies have shown that osteosarcoma cells can spontaneously release exosomes into the surrounding microenvironment, and these exosomes can perform multiple functions, such as inducing or promoting an immune escape of osteosarcoma cells, promoting angiogenesis and the growth of osteosarcoma, as well 
as invasion and metastasis of osteosarcoma. ${ }^{5,6}$ At the same time, tumor cells can secrete exosomes in the form of encapsulation and excretion of anti-tumor drugs, resulting in drug resistance. ${ }^{7,8}$ Therefore, studies on the role of exosomes in the early metastasis and drug resistance mechanisms of osteosarcoma have become the focus of current osteosarcoma research. The detection and analysis of exosomes can not only assist in tumor diagnosis, treatment, and prognosis, but these can also be used as effective carriers of gene preparations or drugs for targeted therapies (Figure 1). After the exosomes have been isolated from the body fluids of tumor patients, high-throughput detection methods, such as genomics and proteomics, were used to select the differentially expressed exosomes between the tumor patients and the normal population, which have a good clinical value for the diagnosis and prognosis assessment of the disease. ${ }^{9-11}$ Since exosomes can be transferred to a target organ, they can be designed as the carriers for precise drug delivery. This way, the loaded drugs can be transported to the target organ, to play a specific therapeutic role. ${ }^{12}$

\section{Biological Functions of Exosomes}

Exosomes are derived from the intracellular body of the cell, which is an organelle formed by intracellular depression. Some intracellular cave-ins give rise to polyvesicles, whose parts are transported to lysosomes, and other parts are transported to the cell surface to fuse with the cell membrane through the regulation of $\mathrm{p} 53 .{ }^{13}$ With the advances in research, it is now believed that exosomes can be secreted by a variety of cells in the physiological and pathological environment, and exist in the supernatant of cultured cells and various body fluids, such as the blood, saliva, urine, breast milk, cerebrospinal fluid, and amniotic fluid. ${ }^{2}$

Exosomes with diameters ranging from 40 to $100 \mathrm{~nm}$ can freely travel between the vascular wall and the stroma. They have membrane transport-associated proteins, integrins, lectins, and immunoglobulin superfamily proteins, among others. MiRNAs, messenger ribonucleic acids (mRNAs), and proteins that are well encapsulated by exosomes have potential as gene therapy vectors. These encapsulated biomacromolecules can play a series of roles in their target cells, for example, exosome-coated mRNA can be expressed in the target cells, while exosome-coated miRNA can inhibit the expression of certain genes. ${ }^{2-4,14,15}$

Exosomes can fuse with the cell membrane of the recipient cells to enter them, and the signal molecules released by exosomes can also be carried into recipient cells to exert specific biological effects. ${ }^{16}$ In addition, proteins or lipid ligands on the surface of exosomes are recognized by and activated by target cell surface receptors, and thus participate in information exchange and signal molecular transduction between local and distant cells. As such, they can regulate cell proliferation, cell differentiation, inflammatory immune response, angiogenesis, tumor metastasis, and drug

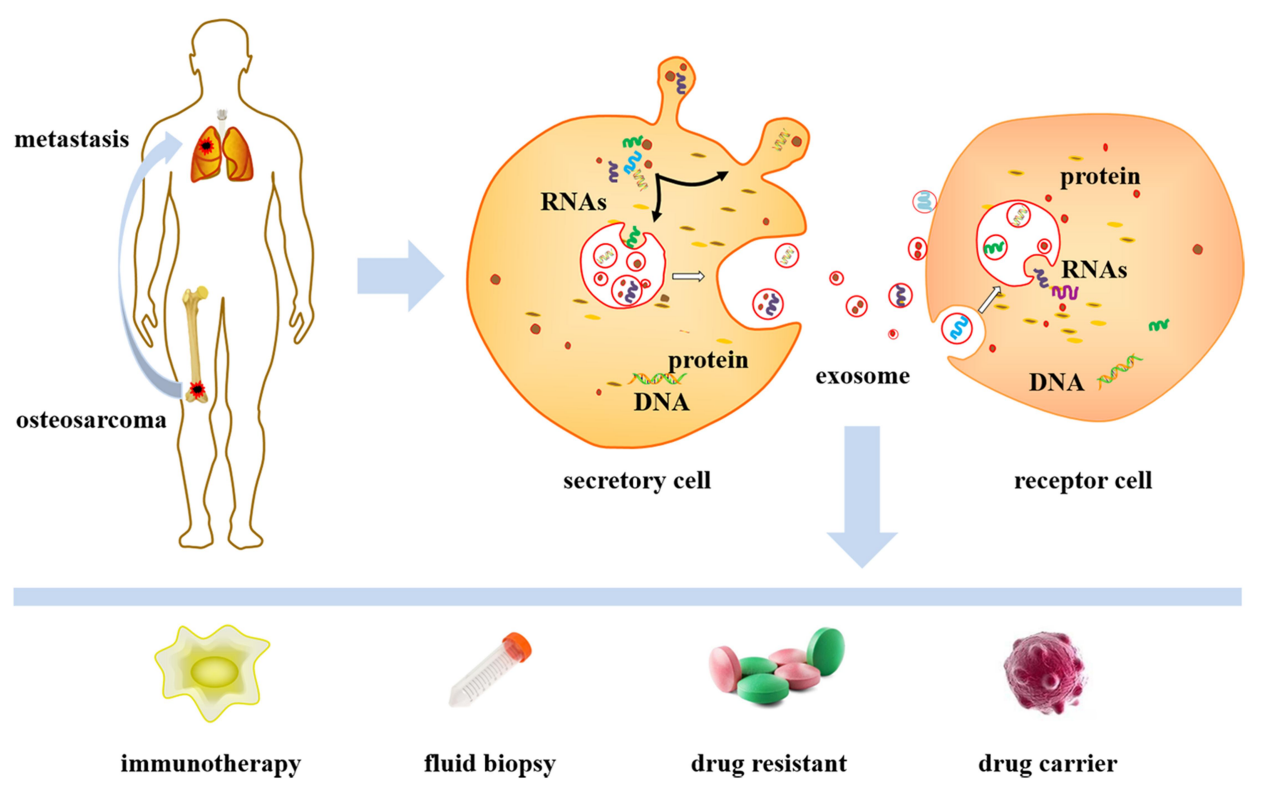

Figure I Exosomes are micro-vesicles with cell-like membrane structures, containing proteins and nucleic acids that participate in intercellular information exchange and regulate cell state and function. Tumor-derived exosomes are closely related to the occurrence, invasion, metastasis, and drug resistance of osteosarcoma. Exosomes have tremendous potential in immunotherapy, liquid biopsy, and multi-drug resistance and may serve as drug carriers for osteosarcoma. 
resistance. ${ }^{17}$ Previous studies have found that exosomes released by tumor cells in the serum of tumor patients carry single-stranded or double-stranded deoxyribonucleic acid (DNA), whose characteristics are highly consistent with those of the DNA found in the primary tumor tissues. Accumulating evidence has shown that the source of exosomes carries a series of bioactive functional molecules with tumor-promoting biological effects. These reactive molecules, including nucleic acids, such as DNA fragments, mRNA, and miRNA; proteins, such as interleukin and integrin cytokines; and lipids. It is estimated that about 19,000, 2838, 27,000, and 1100 kinds of mRNA, miRNAs, proteins, and lipid molecules, respectively, exist in the various types of exosomes. ${ }^{4,5} \mathrm{In}$ addition, the encapsulation of exosomes enables nucleic acid molecules and other substances to stably exist in the extracellular fluid. Therefore, liquid biopsy based on exosomes will open a new chapter of personalized therapies for tumor precision medicine, with great clinical application prospects and research value.

\section{Methods of Exosome Extraction}

There are many methods to extract exosomes, but the most commonly used are overspeed centrifugation, sucrose gradient centrifugation, and immune bead separation (Table 1), with each of them having its own advantages and disadvantages. ${ }^{18}$ At present, there is still no method that takes into consideration the exosome content, purity, or biological activity. Projection electron microscopy, Western blot, and flow cytometry are commonly used to study the biological characteristics of the isolated exosomes, and quantitative reverse transcription-polymerase chain reaction, nucleic acid sequencing, Western blot, or enzyme linked immunosorbent assay are commonly used to determine their function.

Table I Comparison of Exosome Separation Techniques

\begin{tabular}{|c|c|c|c|}
\hline $\begin{array}{l}\text { Separation } \\
\text { Methods }\end{array}$ & Extracting Principle & Advantages & Disadvantages \\
\hline \multirow{2}{*}{$\begin{array}{l}\text { Centrifugal } \\
\text { method } \\
\text { Differential } \\
\text { ultracentrifugation }\end{array}$} & & & \\
\hline & $\begin{array}{l}\text { Continuous separation based on } \\
\text { density, volume, and } \\
\text { morphology of different } \\
\text { substances }\end{array}$ & $\begin{array}{l}\text { Vesicles are concentrated in the } \\
\text { precipitate, with a high yield, and } \\
\text { a large number of samples can be } \\
\text { handled }\end{array}$ & $\begin{array}{l}\text { The equipment is expensive and time- } \\
\text { consuming, the supernatant exosomes are } \\
\text { lost, and the operation is complicated }\end{array}$ \\
\hline $\begin{array}{l}\text { Ultrafiltration } \\
\text { centrifugation }\end{array}$ & $\begin{array}{l}\text { Based on the size difference } \\
\text { between exosomes and other } \\
\text { particles }\end{array}$ & $\begin{array}{l}\text { Low cost, fast extraction speed, no } \\
\text { need for special equipment to } \\
\text { operate }\end{array}$ & $\begin{array}{l}\text { Exosome purity is acceptable, may lead to } \\
\text { exosome loss }\end{array}$ \\
\hline $\begin{array}{l}\text { Sucrose density } \\
\text { gradient } \\
\text { centrifugation }\end{array}$ & $\begin{array}{l}\text { Stratification of low to high } \\
\text { density sediments }\end{array}$ & Exosomes are of high purity & $\begin{array}{l}\text { The preparatory work is complicated and } \\
\text { time-consuming }\end{array}$ \\
\hline $\begin{array}{l}\text { Immunobead } \\
\text { method }\end{array}$ & $\begin{array}{l}\text { The ligand and receptor } \\
\text { connections are performed on } \\
\text { the exosome surface }\end{array}$ & $\begin{array}{l}\text { Exosomes are of high purity and can } \\
\text { be classified }\end{array}$ & $\begin{array}{l}\text { Expensive specific antibodies are required and } \\
\text { the yield is low, suitable for cell-free samples. } \\
\text { Tumor heterogeneity may hamper immune } \\
\text { recognition }\end{array}$ \\
\hline $\begin{array}{l}\text { PEG-based } \\
\text { method }\end{array}$ & $\begin{array}{l}\text { Exosomes with different } \\
\text { solubility and permeability are } \\
\text { separated using a hydrophobic } \\
\text { polymer material }\end{array}$ & $\begin{array}{l}\text { With simple operations, exosomes } \\
\text { can be isolated on a large scale } \\
\text { without special equipment }\end{array}$ & $\begin{array}{l}\text { Exosomes have low purity and the process is } \\
\text { time consuming }\end{array}$ \\
\hline $\begin{array}{l}\text { Chromatography } \\
\text { method }\end{array}$ & $\begin{array}{l}\text { The solute is separated by the } \\
\text { pore size of the gel and the } \\
\text { molecular size of the sample }\end{array}$ & $\begin{array}{l}\text { Exosomes can be extracted quickly, } \\
\text { with low cost, good portability, and } \\
\text { high automation }\end{array}$ & Special equipment is needed \\
\hline $\begin{array}{l}\text { Extraction kit } \\
\text { method }\end{array}$ & $\begin{array}{l}\text { Design based on filtration or } \\
\text { chromatography }\end{array}$ & $\begin{array}{l}\text { Exosomes can be isolated and } \\
\text { extracted without special equipment. } \\
\text { The operation is simple and the } \\
\text { separation is fast }\end{array}$ & $\begin{array}{l}\text { The extraction efficiency is inconsistent with } \\
\text { the purification effect }\end{array}$ \\
\hline
\end{tabular}




\section{Centrifugal Method}

Centrifugal method is a commonly used method for exosome extraction, and the exosomes obtained are large in size but low in purity. ${ }^{19}$ Moreover, the centrifugal force induced by the high speed of the centrifugation can cause changes in the exosome structure and size. The centrifugation methods can be summarized as follows: differential centrifugation, density gradient centrifugation, and ultrafiltration centrifugation.

Differential ultracentrifugation is one of the most classic exosome extraction methods. In the process of centrifugation, low and high-speed alternating centrifugation are used to obtain vesicles with a similar diameter and size. The operation of this method is relatively simple, but the it takes a long time. Repeated centrifugation also destroys the exosomes, which results in low exosome purity and poor treatment. $^{20}$

Ultrafiltration centrifugation is a relatively new extraction method, which uses an ultrafiltration membrane with a different molecular weight cutoff (MWCO) to selectively separate the sample, so that exosomes can be obtained at the end of the process. ${ }^{21}$ This method is simple and efficient and has little effect on the biological activity of exosomes, but the purity of the exosomes obtained is low.

The sucrose density gradient centrifugation method uses a sucrose solution that forms a density layer from low to high after a centrifugation at $100000 \times \mathrm{g}$, so that the exosomes in the sample can be aggregated in the density range of $1.13 \sim 1.19 \mathrm{~g} / \mathrm{mL}$. This enables the obtention of sediments rich in exosomes with high purity; however, the preparation work is complicated and the method is time consuming. ${ }^{22}$

\section{Immunomagnetic Bead Method}

The principle behind this method is that exosomes have specific markers on their surface (such as CD63 and CD9). The globular magnetic particles coated with the monoclonal antibody of one of these markers are used to specifically bind to the exosomes, and exosomes are isolated by the action of a magnetic force. This method ensures the integrity of exosome morphology, high specificity, simplicity, and no expensive equipment required. However, the reactive solution or the magnetic beads wrapped in antibodies may affect the biological activity of the exosomes. Therefore, the choice to opt for this method needs to be in accordance with the design and arrangement of the experiment. $^{23}$

\section{PEG-Based Method}

Polyethylene glycol (PEG) may be competitively bound to free water molecules and can be co-precipitated with hydrophobic proteins and lipids. The extraction of exosomes using the PEG-based method has the following problems: low purity, a low recovery rate, an unequal particle size, more false-positive heteroproteins. Furthermore, the production of difficult-to-remove polymers or added chemicals can destroy the exosomes. ${ }^{24,25}$ All these shortcomings limit the application of the PEG-based method.

\section{Chromatography Method}

The chromatographic method uses the relative relation between the size of the gel pore and the size of the sample to separate the solute, and can also be used to separate exosomes. Observations using an electron microscope show that the exosomes obtained using this method are uniform in size and high in purity. However, this extraction method requires special equipment and is not widely used. ${ }^{26}$

\section{Extraction Kit Method}

Extraction kit is a new exosome extraction method that was developed in recent years. At present, a variety of commercial exosome extraction kits have been designed according to different principles. Some filter out the exosomes using specially designed filters, some isolate and purify the exosomes using space exclusion chromatography, and some precipitate the exosomes using compound precipitation. ${ }^{27,28}$ The extraction kit method does not require special equipment. With the continuous upgrading of products, its extraction efficiency and purification are gradually gaining improvements; thus, it is gradually replacing the overspeed centrifugation method and is currently widely used.

\section{Association Between Exosomes and the Osteosarcoma Microenvironment}

Until now, great research progress has been gradually made in signal transduction pathways related to osteosarcoma, including the role of classical signaling proteins, such as Wnt. Increasing attention has been paid to the potential value of signal transduction in osteosarcoma.

Studies have shown that tumor cells can secrete exosomes and promote tumor growth, metastasis, and angiogenesis by regulating the tumor microenvironment, and even escape from the immune surveillance of tumor cells of the host. $^{2}$ In a previous study, a mouse model of biofluorescent 
osteosarcoma orthotopic transplantation was constructed, and the presence of exosomes in the osteosarcoma microenvironment was detected using transmission electron microscopy. Studies have reported that the vesicles isolated from 143B human osteosarcoma cells contain a variety of molecules with potential effects on the tumor microenvironment, such as matrix metalloproteinases (MMPs), among which MMP-1 and MMP-13 play an important role in extracellular matrix remodeling. ${ }^{29}$ In another study, the researchers have found that the nuclear factor kappa-B (NF- $\mathrm{B}$ ) receptor activator ligand (RANKL) in the exosomes of osteosarcoma cells plays an important role in the activation of MMPs and in the stimulation of osteoclast formation. ${ }^{28}$ Transmembrane 4-superfamily protein CD9 was detected in the tumor microenvironment of 143B human osteosarcoma cells, and CD9 was used as an exosome-specific marker in the ExoCarta database. CD9 is also a membrane fusion protein of precursor osteoclasts, which is involved in the regulation of osteoclast differentiation and maturation. ${ }^{30,31}$ The overexpression of CD9 not only promotes the homing of cancer cells, but also induces osteoclast bone resorption. ${ }^{32}$

The exosomes of osteosarcoma not only regulate the expression and secretion of some cytokines, but also regulate signaling pathways. Wnt ligands or receptors have been shown to be expressed in osteosarcoma cell lines, and the differentiation potential of osteosarcoma cells may be highly correlated with the autocrine expression of Wnt signaling pathway. ${ }^{33}$ Kansara et al found that after targeted inhibition of the expression of Wnt inhibitor 1 (Wif 1), the incidence of osteosarcoma in experimental mice significantly increased, suggesting that the activation of Wnt signaling pathway may promote the occurrence of osteosarcoma. ${ }^{34}$ Studies have found that the overexpression of CD82 and CD9 can induce cells to secrete exosomes and excrete cell-catenin, thus significantly inhibiting the signaling activity of Wnt. ${ }^{35}$ It can be seen that exosomes extensively participate in the regulation of various components of the Wnt signaling pathway, directly or indirectly affecting cell function. In addition, it has also been found that transforming growth factor- $\beta$ (TGF$\beta 1)$ in tumor exosomes can significantly down-regulate the expression of Natural killer group 2 member D (NKG2D) on the surface of CD8 $(+)$ T cells and natural killer (NK) cells, thereby reducing the activation of lymphocytes and the immune recognition of tumor cells. Therefore, it is proposed that NKG2D can be used as a physiological indicator for exosomes to regulate tumor immune evasion. ${ }^{36}$

With the research advances on the signaling pathways, targeted drugs with lower dose and less toxicity have been developed, as they are able to act more effectively on key targets of the corresponding signaling pathway. However, the occurrence and development of osteosarcoma involves the interaction of multiple signaling pathways, and only a thorough study of this correlation can more effectively improve the treatment of osteosarcoma.

\section{The Role of Exosomes in Distant Metastasis of Osteosarcoma}

Osteosarcomas are often accompanied by distant metastases, and it has been found that exosomes, as extracellular vesicles, play an important role in the spread of tumors. Exosomes have been proven to be important in metastasis organotropism, induction of angiogenesis, and vascular permeability, as well as in the education of cells towards a pro-metastatic phenotype or in the interaction between stromal and tumor cells. Tumor-derived exosomes regulate the metastasis of tumor cells in the following three aspects: ${ }^{37,38}$ tumor cells directly induce tumor metastasis by secreting exosomes; tumor-derived exosomes mediated by miRNAs regulate the microenvironment of tumor cell growth; tumor-derived exosomes indirectly promote tumor metastasis by transforming distant mesenchymal cells.

MiRNA can be transported from tissue to tissue, as genetic material, via exosome inclusion. In a recent study, gene chip technology has been used to detect the miRNAs isolated from osteosarcomas and osteoblasts with a different metastatic potential, and they found that the most prominent miRNAs were miR-21-5p, miR-143-3p, miR-148a-3p, and $181 \mathrm{a}-5 \mathrm{p}$, whose expression levels were higher in metastatic SAOS2 cells, compared to non-metastatic MG63 cells. Further bioinformatics studies have shown that miRNAs may regulate metastasis potential through mitogenactivated protein kinase 1 (MAPK1), neuroblastoma Ras (NRAS), fibroblast growth factor receptor substrate 2 (FRS2), B-cell lymphoma 2 (BCL2), and Quaking (QKI). ${ }^{39}$ Another study also showed that miR-675 in serum exosomes is associated with osteosarcoma, and its high expression may be a new biomarker for osteosarcoma metastasis. ${ }^{40}$ MiR-143 has a negative regulatory effect on human osteosarcoma cells. Studies have found that miR-143 can inhibit the lung metastasis of osteosarcoma after implantation in a mouse tumor model, and that the up-regulation of miR-143 can significantly reduce cell activity, promote apoptosis, and inhibit tumor cell growth. ${ }^{41,42}$ A study on osteosarcoma specimens showed that TGF- $\beta 1$ could enhance the expression of the proteoglycan by inhibiting miR-143, thus 
enhancing the invasion and metastasis of osteosarcoma. ${ }^{43}$ Derived from cancer cells, miR-21 contained within exosomes in the tumor microenvironment may act on both cancer cells and the surrounding tumor microenvironment (TME), including immune cells, endothelial cells, and fibroblasts. In human serum and plasm, the level of exosomal miR-21 between osteosarcoma patients and healthy controls differs, supporting the role of miR-21 as an osteosarcoma biomarker. The involvement of a number of miR-21 target genes in tumor progression suggests that miR-21 may significantly affect the plasticity of cancer cells, leading to tumor progression, metastasis, angiogenesis, and immune escape in osteosarcoma. ${ }^{44}$

Hooper et al found that Wnt signaling can induce exosome secretion, and that the components of the Wnt signaling pathway can act on distant cells. ${ }^{45}$ Another study found that high concentrations on the edge of osteosarcoma cells expressing Dkk-1 (Wnt signal inhibitor) and its excretion may be associated with tumor invasiveness through the Dkk-1 antibody blocking effect. This effect can significantly inhibit osteogenesis and sarcoma cell proliferation of MG63 cells. It is speculated that bones are necessary for the normal development of the inactivation of the Wnt signaling pathway, which may be associated with osteosarcoma occurrence. ${ }^{46}$ These studies on the exosomes that are secreted by osteosarcoma cells and the Wnt signaling pathway provide a theoretical basis for targeted osteosarcoma therapy.

TGF- $\beta$ plays an important role in tumor invasion and metastasis, cell proliferation and differentiation, extracellular matrix production, angiogenesis, cell apoptosis, and regulation of the immune function. TGF- $\beta$ expression has been detected in osteosarcoma cell exosomes, which can directly or indirectly regulate the secretion of chemokine ligand 16 (CXCL16) via osteoclasts, in order to regulate the metastasis of osteoblastic precursor cells and osteosarcoma cells. $^{30}$ At the same time, TGF- $\beta$ induces the differentiation of monocytes and the accumulation of immature myeloid suppressor cells (MDSCs), ${ }^{47}$ and MDSCs have been shown to promote osteoclast bone resorption in the tumor microenvironment. ${ }^{48}$

\section{The Role of Exosomes in Chemotherapy Resistance of Osteosarcoma}

At present, the main treatment for osteosarcoma is neoadjuvant chemotherapy combined with surgery. Although most osteosarcoma patients are sensitive to the initial chemotherapy, some patients still present resistance to chemotherapy. In recent years, many studies have shown that exosomes also play an important regulatory role in chemotherapy resistance of osteosarcoma. ${ }^{49}$

Studies have confirmed a positive correlation between tumor cell resistance to docetaxel and exosome secretion. $^{50}$ Exosome encapsulation and excretion of a variety of different anti-tumor drugs have been captured using fluorescence microscopy, in a variety of tumors and may be related to the sensitivity of anti-tumor drugs. ${ }^{51}$ Chemotherapy drugs are excreted through exosomes, which are secreted by tumor cells, and thus, antibodybased targeting drugs can be neutralized.

In a study, the potential role of exosomes to transfer their multidrug resistance (MDR) phenotype in human osteosarcoma cells was investigated. They suggested that multidrug resistant osteosarcoma cells can spread the ability to resist to doxorubicin onto sensitive cells by transferring exosomes carrying MDR-1 mRNA and P-glycoprotein. ${ }^{6}$ Another study analyzed the microRNA and mRNA whose expression in exosomes in an osteosarcoma context can predict the response to chemotherapy. They found that exosomal RNA can be considered as a reliable biomarker to predict the sensitivity and response to chemotherapy in osteosarcoma. It was shown that, using osteosarcoma cell-derived exosomes, miR-135b and other RNAs that could render osteosarcoma resistant to chemotherapy drugs could be isolated. ${ }^{52}$ At the same time, exosomes can also play the role of delivering glycoproteins, making osteosarcoma resistant to chemotherapy drugs. For example, during the delivery of glycoproteins by exosomes, osteosarcoma cells can obtain anti-tumor drug substances from other tumor cells to achieve resistance to paclitaxel. ${ }^{53}$

Chemotherapy is an important part of the treatment of osteosarcoma. To improve the chemotherapy effects on osteosarcoma patients, it is particularly important to explore the specific mechanisms of inclusion, transportation, and excretion of chemotherapeutic drugs and drug metabolites in exosomes. Further studies on exosomes may lead to the finding of new targets to block chemotherapy resistance.

\section{The Role of Exosomes in Diagnosis and Prognostic Evaluation of Osteosarcoma}

For mesenchymal tissue-derived osteosarcoma, due to the apparent tumor heterogeneity and lack of classical tumor 
markers, the cell marker detection role of circulating tumor cells in the prognostic monitoring of mesenchymal tissue-derived malignancies - such as osteosarcoma - is significantly restricted. As a new way of liquid biopsy, exosomes of tumor cells in body fluids have attracted much attention because they carry some functional proteins and genes of tumor cells. An early diagnosis and early standardized treatment of osteosarcoma have always been the ideal clinical goals. Recent exosome studies have proved that miRNAs may be useful in the early diagnosis of osteosarcoma. However, RNAs in the blood are highly unstable and are easily degraded, which makes it difficult to detect the relevant miRNA levels in circulation. However, the membrane of exosomes can play a protective role, rendering miRNAs in exosomes relatively stable, and thus increasing the potential effectiveness of the clinical application of miRNA. ${ }^{54-56}$

It was reported that MMPs can be used as tumor markers to evaluate the prognosis of osteosarcoma. ${ }^{29}$ A study confirmed that the Wnt-related receptor lipoprotein receptorrelated protein 5 (LRP5) can be used as a marker for the disease progression of osteosarcoma, and emphasized the important role of LRP5 and Wnt signaling pathways in the pathology and disease development of osteosarcoma. ${ }^{57}$ In a recent study, researchers have found that the preoperative and postoperative serum levels of basic fibroblast growth factor (bFGF) in osteosarcoma patients were significantly higher than those in normal controls, and that the bFGF level could effectively evaluate the disease progression and prognosis of osteosarcoma. In vitro experiments have shown that the exosome delivery of TGF- $\beta 1$ inhibits interleukin 2 (IL-2) induced lymphocyte proliferation, thereby promoting the immune escape of tumor cells, and that its inhibitory efficiency is 1400 times higher than that of soluble TGF- $\beta 1{ }^{58}$

The characteristic molecules of the exosome membrane structure can reflect the phenotype of the tumor of origin, and the exosomes in preoperative and postoperative peripheral blood can be used as an important indicator of early diagnosis and prognosis. Exosome detection is an important part of fluid biopsy and should be paid more attention by scholars. Compared with malignant tumor specimens, tumor-derived exosomes are more easily obtained from body fluids (including serum, plasma, and urine), so they may be of important significance in the diagnosis and prognosis of osteosarcoma. ${ }^{59}$ A study involving 48 osteosarcoma patients indicated that miRNAs in exosomes have the ability to assess a patient's response to chemotherapy. Eight miRNAs, including miR-148a, miR133, and miR-27a are expected to be biomarkers for osteosarcoma patients with a poor chemotherapy response. These miRNAs are also present in the exosomes of the blood of patients, and their content is comparable to that of tumor cells. In addition, these miRNAs can also be isolated and extracted from other body fluids, which indicates that exosomes can be used as markers to diagnose cancer, as well as important indicators of changes in the condition and prognosis of cancer patients. ${ }^{52}$

\section{The Role of Exosomes in the Treatment of Osteosarcoma}

Different tumor-derived exosomes carry different surface proteins, and high-throughput detection techniques are used to search for characteristic proteins between tumor patients and normal controls, which is helpful for the diagnosis of diseases and the assessment of prognosis. At the same time, specific proteins, screened based on proteomics, can identify whether exosomes come from normal or tumor cells. Since exosomes can be transferred to target organs, they can be used as carriers for an accurate drug delivery to a target organ to play an anti-tumor effect. It can be seen that exosomes display great application potential as drug targeting vectors.

It was found that after an artificial miR-143 was introduced into bone mesenchymal stem cells (BMSCs), it acted on osteosarcoma cells in the form of exosome encapsulation to reduce the metastasis ability of tumor cells, and it was confirmed that exosome miRNA transport was more effective than other transfection methods in intercellular transport, related gene expression, and cell function. ${ }^{60}$ Therefore, the use of exosomes to package miRNAs on tumor cells has shown advantages in the treatment of osteosarcoma. The membranous structure of tumorderived exosomes is rich in tumor-related antigens, major histocompatibility complex molecules, and co-stimulators, which can induce a toxic T-cell immune response of antitumor cells. ${ }^{61}$ A recent study has found that the combination of dendritic cells and anti-TGF- $\beta$ antibody not only inhibits the proliferation of osteosarcoma cells, but also activates the systemic immune system to improve the therapeutic effect of osteosarcoma. ${ }^{62}$ In an anti-tumor drugs study, a nano-drug consisting of doxorubicin and exosomes derived from MSCs (Exo-Dox) has been developed, and its efficiency as a chemotherapeutic drug for the treatment of osteosarcoma in vitro has been explored. 
Considering the tumor-homing feature of BM-MSCs, the Exo-Dox can be an impressive choice for targeted osteosarcoma treatment in the future. ${ }^{63}$

\section{Conclusion}

Exosomes, which are ubiquitous nanoscale membrane structures in the body, are widely involved in intercellular communication. Exosomes have been the focus of research in regard to the physiological and pathological processes of the organism. With the increasing attention, and the increasingly mature analytical methods of high sensitivity, high throughput, and high connotation, increasing amounts of information have been obtained on the transfer function of exosomes. Exosome related research has entered a new stage, and its clinical application has become a research hotspot in recent years. Several studies have shown that exosomes can not only regulate the proliferation and apoptosis of malignant tumor cells, but also affect the microenvironment of tumor cell growth, thus affecting the occurrence, development, metastasis, immune escape, and chemotherapy resistance of malignant tumors.

In the field of osteosarcoma, due to the characteristics of tumor tissues and the number of samples and other factors, exosome-related studies are lagging behind, and clinical studies on the application of exosomes need to be carried out in the future. Exosomes can promote the angiogenesis of osteosarcoma by regulating the Wnt, TGF- $\beta$, and characterization of human calneuron 1 (CALN1) signaling pathways. In addition, osteosarcoma-derived exosomes that are rich in MMPs and a disintegrin and metalloproteinases (ADAMs) can promote the invasion and metastasis of osteosarcoma. In addition, some exosome-delivered miRNAs and ATP-binding box transporters (ABCs) may also be regulatory factors of increased tumor drug resistance. In conclusion, exosomes can promote the occurrence, invasion, metastasis, immune escape, and drug resistance of osteosarcoma, and play an important role in the diagnosis and treatment of osteosarcoma.

The characteristic molecules of the exosome membrane structure can reflect the phenotype of the tumor, and the detection of exosomes in the peripheral blood of osteosarcoma patients before and after surgery can be used as an important indicator for early diagnosis and prognosis of osteosarcoma. At present, chemotherapy for osteosarcoma has entered a plateau, and the mechanism of exosome packaging and excretion of anti-tumor cells is being further studied. This mode of intercellular transport can effectively avoid host immune response; thus, exosomes can be used as carriers to transport drugs, which can be biological or gene therapy agents to targeted sites. In the future, according to the characteristics of exosomes that can be modified and processed, a new way of thinking will be provided for the early diagnosis, treatment, and development of new anti-tumor drugs for osteosarcoma.

\section{Funding}

The present study was supported by The National Natural Science Foundation of China (grant no. 81904231), the China Postdoctoral Science Foundation (grant no. 2020M672369), and the PostDoctoral Innovation Practice Post in Hubei Province (grant no. 34).

\section{Disclosure}

The authors report no conflicts of interest in this work.

\section{References}

1. Ferrari S, Serra M. An update on chemotherapy for osteosarcoma. Expert Opin Pharmacother. 2015;16(18):2727-2736. doi:10.1517/ 14656566.2015.1102226

2. Xie F, Zhou X, Fang M, et al. Extracellular vesicles in cancer immune microenvironment and cancer immunotherapy. $A d v S c i$ (Weinh). 2019;6(24):1901779. doi:10.1002/advs.201901779

3. Melo SA, Sugimoto H, O'Connell JT, et al. Cancer exosomes perform cell-independent microRNA biogenesis and promote tumorigenesis. Cancer Cell. 2014;26(5):707-721. doi:10.1016/j. ccell.2014.09.005

4. Milane L, Singh A, Mattheolabakis G, et al. Exosome mediated communication within the tumor microenvironment. J Control Release. 2015;219:278-294. doi:10.1016/j.jconrel.2015.06.029

5. Mu X, Agarwal R, March D, et al. Notch signaling mediates skeletal muscle atrophy in cancer cachexia caused by osteosarcoma. Sarcoma. 2016;2016:3758162. doi:10.1155/2016/3758162

6. Torreggiani E, Roncuzzi L, Perut F, et al. Multimodal transfer of MDR by exosomes in human osteosarcoma. Int J Oncol. 2016;49 (1):189-196. doi:10.3892/ijo.2016.3509

7. Daßler-Plenker J, Küttner V, Egeblad M. Communication in tiny packages: exosomes as means of tumor-stroma communication. Biochim Biophys Acta Rev Cancer. 2020;1873(2):188340. doi:10. 1016/j.bbcan.2020.188340

8. Zhang YF, Shi JB, Li C. Small extracellular vesicle loading systems in cancer therapy: current status and the way forward. Cytotherapy. 2019;21(11):1122-1136. doi:10.1016/j.jcyt.2019.10.002

9. de Carvalho IN, de Freitas RM, Vargas FR. Translating microRNAs into biomarkers: what is new for pediatric cancer? Med Oncol. 2016;33(5):49. doi:10.1007/s12032-016-0766-4

10. Galardi A, Colletti M, Di Paolo V, et al. Exosomal MiRNAs in pediatric cancers. Int J Mol Sci. 2019;20(18):4600. doi:10.3390/ ijms 20184600

11. Troyer RM, Ruby CE, Goodall CP, et al. Exosomes from osteosarcoma and normal osteoblast differ in proteomic cargo and immunomodulatory effects on T cells. Exp Cell Res. 2017;358(2):369-376. doi:10.1016/j.yexcr.2017.07.011

12. Birru B, Durthi CP, Kacham S, et al. Stem cells in tumour microenvironment aid in prolonged survival rate of cancer cells and developed drug resistance: major challenge in osteosarcoma treatment. Curr Drug Metab. 2020;21(1):44-52. doi:10.2174/138920022 1666200214120226 
13. Azulay EE, Cooks T, Elkabets M. Potential oncogenic roles of mutant-p53-derived exosomes in the tumor-host interaction of head and neck cancers. Cancer Immunol Immunother. 2020;69(2):285-292.

14. Théry C, Witwer KW, Aikawa E, et al. Minimal information for studies of extracellular vesicles 2018 (MISEV2018): a position statement of the international society for extracellular vesicles and update of the MISEV2014 guidelines. J Extracell Vesicles. 2018;7(1):1535750.

15. Lötvall J, Hill AF, Hochberg F, et al. Minimal experimental requirements for definition of extracellular vesicles and their functions: a position statement from the international society for extracellular vesicles. J Extracell Vesicles. 2014;3:26913. doi:10.3402/jev.v3.26913

16. Frydrychowicz M, Kolecka-Bednarczyk A, Madejczyk M, et al. Exosomes - structure, biogenesis and biological role in non-small-cell lung cancer. Scand J Immunol. 2015;81(1):2-10. doi:10.1111/sji.12247

17. Qin J, Xu Q. Functions and application of exosomes. Acta Pol Pharm. 2014;71(4):537-543.

18. Chen BY, Sung CW, Chen C, et al. Advances in exosomes technology. Clin Chim Acta. 2019;493:14-19. doi:10.1016/j. cca.2019.02.021

19. Petersen KE, Manangon E, Hood JL, et al. A review of exosome separation techniques and characterization of B16-F10 mouse melanoma exosomes with AF4-UV-MALS-DLS-TEM. Anal Bioanal Chem. 2014;406(30):7855-7866. doi:10.1007/s00216-014-8040-0

20. Livshits MA, Khomyakova E, Evtushenko EG, et al. Isolation of exosomes by differential centrifugation: theoretical analysis of a commonly used protocol. Sci Rep. 2015;5:17319. doi:10.1038/srep17319

21. Xu R, Simpson RJ, Greening DW. A protocol for isolation and proteomic characterization of distinct extracellular vesicle subtypes by sequential centrifugal ultrafiltration. Methods Mol Biol. 2017;1545:91-116.

22. Stanly C, Fiume I, Capasso G, et al. Isolation of exosome-like vesicles from plants by ultracentrifugation on sucrose/deuterium oxide (D2O) density cushions. Methods Mol Biol. 2016;1459:259-269.

23. Oksvold MP, Neurauter A, Pedersen KW. Magnetic bead-based isolation of exosomes. Methods Mol Biol. 2015;1218:465-481.

24. Weng Y, Sui Z, Shan Y, et al. Effective isolation of exosomes with polyethylene glycol from cell culture supernatant for in-depth proteome profiling. Analyst. 2016;141(15):4640-4646. doi:10.1039/ C6AN00892E

25. Ludwig AK, De Miroschedji K, Doeppner TR, et al. Precipitation with polyethylene glycol followed by washing and pelleting by ultracentrifugation enriches extracellular vesicles from tissue culture supernatants in small and large scales. J Extracell Vesicles. 2018;7 (1):1528109. doi:10.1080/20013078.2018.1528109

26. Singhto N, Vinaiphat A, Thongboonkerd V. Discrimination of urinary exosomes from microvesicles by lipidomics using thin layer liquid chromatography (TLC) coupled with MALDI-TOF mass spectrometry. Sci Rep. 2019;9(1):13834. doi:10.1038/s41598-019-50195-Z

27. Chen P, Ruan A, Zhou J, et al. Extraction and identification of synovial tissue-derived exosomes by different separation techniques. J Orthop Surg Res. 2020;15(1):97. doi:10.1186/s13018-020-01604-x

28. Tang YT, Huang YY, Zheng L, et al. Comparison of isolation methods of exosomes and exosomal RNA from cell culture medium and serum. Int $J$ Mol Med. 2017;40(3):834-844. doi:10.3892/ ijmm.2017.3080

29. Garimella R, Eskew J, Bhamidi P, et al. Biological characterization of preclinical Bioluminescent Osteosarcoma Orthotopic Mouse (BOOM) model: a multi-modality approach. J Bone Oncol. 2013;2 (1):11-21. doi:10.1016/j.jbo.2012.12.005

30. Ota K, Quint P, Weivoda MM, et al. Transforming growth factor beta 1 induces CXCL16 and leukemia inhibitory factor expression in osteoclasts to modulate migration of osteoblast progenitors. Bone. 2013;57(1):68-75. doi:10.1016/j.bone.2013.07.023

31. Ishii M, Iwai K, Koike $M$, et al. RANKL-induced expression of tetraspanin CD9 in lipid raft membrane microdomain is essential for cell fusion during osteoclastogenesis. $J$ Bone Miner Res. 2006;21(6):965-976. doi:10.1359/jbmr.060308
32. Kischel P, Bellahcene A, Deux B, et al. Overexpression of CD9 in human breast cancer cells promotes the development of bone metastases. Anticancer Res. 2012;32(12):5211-5220.

33. Chen C, Zhao M, Tian A, et al. Aberrant activation of Wnt/ $\beta$-catenin signaling drives proliferation of bone sarcoma cells. Oncotarget. 2015;6(19):17570-17583. doi:10.18632/oncotarget.4100

34. Kansara M, Tsang M, Kodjabachian L. Wnt inhibitory factor 1 is epigenetically silenced in human osteosarcoma, and targeted disruption accelerates osteosarcomagenesis in mice. J Clin Invest. 2009;119 (4):837-851. doi:10.1172/JCI37175

35. Chairoungdua A, Smith DL, Pochard P, et al. Exosome release of $\beta$ catenin: a novel mechanism that antagonizes Wnt signaling. $J$ Cell Biol. 2010;190(6):1079-1091. doi:10.1083/jcb.201002049

36. Clayton A, Mitchell JP, Court J, et al. Human tumor-derived exosomes down-modulate NKG2D expression. J Immunol. 2008;180 (11):7249-7258. doi:10.4049/jimmunol.180.11.7249

37. Tauro BJ, Mathias RA, Greening DW, et al. Oncogenic H-ras reprograms Madin-Darby canine kidney (MDCK) cell-derived exosomal proteins following epithelial-mesenchymal transition. Mol Cell Proteomics. 2013;12(8):2148-2159. doi:10.1074/mcp.M112.027086

38. Kogure T, Lin W-L, Yan IK, et al. Intercellular nanovesicle-mediated microRNA transfer: a mechanism of environmental modulation of hepatocellular cancer cell growth. Hepatology. 2011;54 (4):1237-1248. doi:10.1002/hep.24504

39. Jerez S, Araya H, Hevia D, et al. Extracellular vesicles from osteosarcoma cell lines contain miRNAs associated with cell adhesion and apoptosis. Gene. 2019;710:246-257. doi:10.1016/j.gene.2019.06.005

40. Gong L, Bao Q, Hu C, et al. Exosomal miR-675 from metastatic osteosarcoma promotes cell migration and invasion by targeting CALN1. Biochem Biophys Res Commun. 2018;500(2):170-176. doi:10.1016/j.bbrc.2018.04.016

41. Osaki M, Takeshita F, Sugimoto Y, et al. MicroRNA-143 regulates human osteosarcoma metastasis by regulating matrix metalloprotease-13 expression. Mol Ther. 2011;19(6):1123-1130. doi:10.1038/mt.2011.53

42. Li W-H, Wu H-J, Li Y-X, et al. MicroRNA-143 promotes apoptosis of osteosarcoma cells by caspase-3 activation via targeting Bcl-2. Biomed Pharmacother. 2016;80:8-15. doi:10.1016/j.biopha.2016.03.001

43. Li F, Li S, Cheng T. TGF- $\beta 1$ promotes osteosarcoma cell migration and invasion through the miR-143-versican pathway. Cell Physiol Biochem. 2014;34(6):2169-2179. doi:10.1159/000369660

44. Wang S, Ma F, Feng Y, et al. Role of exosomal miR-21 in the tumor microenvironment and osteosarcoma tumorigenesis and progression (Review). Int J Oncol. 2020;56(5):1055-1063. doi:10.3892/ ijo.2020.4992

45. Hooper C, Sainz-Fuertes R, Lynham S, et al. Wnt3a induces exosome secretion from primary cultured rat microglia. BMC Neurosci. 2012;13:144. doi:10.1186/1471-2202-13-144

46. Lee N, Smolarz AJ, Olson S, et al. A potential role for Dkk-1 in the pathogenesis of osteosarcoma predicts novel diagnostic and treatment strategies. Br J Cancer. 2007;97(11):1552-1559. doi:10.1038/sj. bjc. 6604069

47. Xiang X, Poliakov A, Liu C, et al. Induction of myeloid-derived suppressor cells by tumor exosomes. Int J Cancer. 2009;124 (11):2621-2633. doi:10.1002/ijc.24249

48. Danilin S, Merkel AR, Johnson JR, et al. Myeloid-derived suppressor cells expand during breast cancer progression and promote tumor-induced bone destruction. Oncoimmunology. 2012;1 (9):1484-1494. doi:10.4161/onci.21990

49. Bielack SS, Hecker-Nolting S, Blattmann C, et al. Advances in the management of osteosarcoma.F1000Res. 2016;5:2767. doi:10.12688/ f1000research.9465.1

50. Corcoran C, Rani S, O'Brien K, et al. Docetaxel-resistance in prostate cancer: evaluating associated phenotypic changes and potential for resistance transfer via exosomes. PLoS One. 2012;7(12):e50999. doi:10.1371/journal.pone.0050999 
51. Shedden K, Xie XT, Chandaroy P, et al. Expulsion of small molecules in vesicles shed by cancer cells: association with gene expression and chemosensitivity profiles. Cancer Res. 2003;63 (15):4331-4337.

52. Xu JF, Wang YP, Zhang SJ, et al. Exosomes containing differential expression of microRNA and mRNA in osteosarcoma that can predict response to chemotherapy. Oncotarget. 2017;8 (44):75968-75978. doi:10.18632/oncotarget.18373

53. Yu DD, Wu Y, Shen HY, et al. Exosomes in development, metastasis and drug resistance of breast cancer. Cancer Sci. 2015;106 (8):959-964. doi:10.1111/cas.12715

54. Li B, Song Y, Liu TJ, et al. miRNA-22 suppresses colon cancer cell migration and invasion by inhibiting the expression of T-cell lymphoma invasion and metastasis 1 and matrix metalloproteinases 2 and 9. Oncol Rep. 2013;29(5):1932-1938. doi:10.3892/or.2013.2300

55. Gao Q, Lei F, Zeng Q, et al. Functional passenger-strand miRNAs in exosomes derived from human colon cancer cells and their heterogeneous paracrine effects. Int J Biol Sci. 2020;16(6):1044-1058. doi:10.7150/ijbs.40787

56. Hannafon BN, Trigoso YD, Calloway CL, et al. Plasma exosome microRNAs are indicative of breast cancer. Breast Cancer Res. 2016;18(1):90. doi:10.1186/s13058-016-0753-x

57. Hoang BH, Kubo T, Healey $\mathrm{JH}$, et al. Expression of LDL receptor-related protein 5 (LRP5) as a novel marker for disease progression in high-grade osteosarcoma. Int J Cancer. 2004;109 (1):106-111. doi:10.1002/ijc.11677
58. Clayton A, Mitchell JP, Court J, et al. Human tumor-derived exosomes selectively impair lymphocyte responses to interleukin-2. Cancer Res. 2007;67(15):7458-7466. doi:10.1158/0008-5472.CAN06-3456

59. Grimaldi A, Zarone MR, Irace C, et al. Non-coding RNAs as a new dawn in tumor diagnosis. Semin Cell Dev Biol. 2018;78:37-50. doi:10.1016/j.semcdb.2017.07.035

60. Shimbo K, Miyaki S, Ishitobi H, et al. Exosome-formed synthetic microRNA-143 is transferred to osteosarcoma cells and inhibits their migration. Biochem Biophys Res Commun. 2014;445(2):381-387. doi:10.1016/j.bbrc.2014.02.007

61. Tauro BJ, Greening DW, Mathias RA, et al. Two distinct populations of exosomes are released from LIM1863 colon carcinoma cell-derived organoids. Mol Cell Proteomics. 2013;12(3):587-598. doi:10.1074/mcp.M112.021303

62. Kawano M, Itonaga I, Iwasaki T, et al. Anti-TGF- $\beta$ antibody combined with dendritic cells produce antitumor effects in osteosarcoma. Clin Orthop Relat Res. 2012;470(8):2288-2294. doi:10.1007/s11999012-2299-2

63. Wei H, Chen J, Wang S, et al. A nanodrug consisting of doxorubicin and exosome derived from mesenchymal stem cells for osteosarcoma treatment in vitro. Int $J$ Nanomedicine. 2019;14:8603-8610. doi:10.2147/IJN.S218988

\section{Publish your work in this journal}

OncoTargets and Therapy is an international, peer-reviewed, open access journal focusing on the pathological basis of all cancers, potential targets for therapy and treatment protocols employed to improve the management of cancer patients. The journal also focuses on the impact of management programs and new therapeutic agents and protocols on patient perspectives such as quality of life, adherence and satisfaction. The manuscript management system is completely online and includes a very quick and fair peer-review system, which is all easy to use. Visit http://www.dovepress.com/ testimonials.php to read real quotes from published authors. 